\title{
PF4-Dependent Immunoassays in Patients with Vaccine-Induced Immune Thrombotic Thrombocytopenia: Results of an Interlaboratory Comparison
}

\author{
Ulrich J. Sachs ${ }^{1,2}$ Nina Cooper ${ }^{1,2}$ Andreas Czwalinna ${ }^{3}$ Jens Müller ${ }^{4}$ Bernd Pötzsch ${ }^{4}$ \\ Andreas Tiede ${ }^{5, *([)}$ Karina Althaus $6, *(0)$ \\ ${ }^{1}$ Department of Thrombosis and Haemostasis, Giessen University \\ Hospital, Giessen, Germany \\ 2 Institute for Clinical Immunology and Transfusion Medicine, Justus \\ Liebig University, Giessen, Germany \\ ${ }^{3}$ Amedes WagnerStibbe Medical Laboratory, Hannover, Germany \\ ${ }^{4}$ Institute for Experimental Haematology and Transfusion Medicine, \\ University of Bonn, Bonn, Germany \\ ${ }^{5}$ Department of Haematology, Haemostasis, Oncology, and Stem Cell \\ Transplantation, Hannover Medical School, Hannover, Germany \\ Address for correspondence Karina Althaus, MD, Institute for Clinical \\ and Experimental Transfusion Medicine, Medical Faculty of \\ Tuebingen, Otfried-Müller-Str. 4/1, 72076 Tübingen, Germany \\ (e-mail: karina.althaus@med.uni-tuebingen.de; \\ ulrich.sachs@med.uni-giessen.de). \\ ${ }^{6}$ Institute for Clinical and Experimental Transfusion Medicine, Medical \\ Faculty of Tuebingen, University Hospital of Tuebingen, Tuebingen, \\ Germany
}

Thromb Haemost 2021;121:1622-1627.

\begin{abstract}
Keywords

- COVID-19 vaccine

- thrombosis

- antibody

- immunoassay

- platelet factor 4

Background Coronavirus disease 2019 vaccine ChAdOx 1 nCov-19 may rarely lead to vaccine-induced thrombotic thrombocytopenia (VITT). Antibody-mediated, platelet factor 4 (PF4)-dependent platelet activation appears to resemble a key mechanism in VITT, partially comparable to heparin-induced thrombocytopenia. The use of PF4/ heparin immunoassays has been proposed as part of a diagnostic approach, but their sensitivity has not been established.

Methods Sera from 12 well-defined VITT patients were first studied by two different laboratories in functional assays. Sera where then used for an interlaboratory comparison, in which five different PF4/heparin immunoassays were used by four laboratories. Results Results for functional testing were highly concordant. VITT antibodies were also reliably detected by PF4/heparin enzyme-linked immunosorbent assays (ELISAs) (92-100\%). In contrast, only $25 \%$ of VITT antibodies were reactive in a particle gel immunoassay (PaGIA), and $8 \%$ in a lateral flow assay (LFA). An automated chemiluminescence immunoassay (CLIA) was negative for all sera tested $(0 \%)$.

Conclusion It seems feasible to establish functional antibody testing for the confirmation of VITT. For the initial screening of suspected VITT cases, PaGIA, LFA, and CLIA are useless when applied as single tests. Only ELISA-based PF4/heparin immunoassays are sensitive enough to be incorporated in the diagnostic workup. However, a combination of a positive ELISA and a negative CLIA may be useful to identify VITT antibodies in the absence of confirmatory functional assays.
\end{abstract}

These authors contributed equally to this study.

received

May 15, 2021

accepted after revision

June 22, 2021

published online

June 24, 2021 (c) 2021. Thieme. All rights reserved. Georg Thieme Verlag KG,

Rüdigerstraße 14,

70469 Stuttgart, Germany
DOI https://doi.org/

10.1055/a-1535-9002.

ISSN 0340-6245. 


\section{Introduction}

The coronavirus disease 2019 (COVID-19) vaccine ChAdOx1 nCov-19 (AZD1222, Vaxzevria) was recently associated with the development of vaccine-induced thrombotic thrombocytopenia (VITT). In the majority of cases thrombocytopenia occurred within 5 to 16 days after vaccination with ChAdOx 1 nCov-19 and were associated with the development of thrombosis. ${ }^{1-4}$ Patients were between 22 and 67 years of age. Thrombotic sites included the cerebral sinus, cortical and cerebral veins, splanchnic veins, and the right ventricle. Microvascular manifestations in the brain, the lungs, and the kidneys were also seen. Three patients presented with pulmonary embolism. Arterial manifestations included stroke, aortic thrombosis, and limb artery thrombosis. In all patients, platelet counts were low, between 10,000 and $107,000 / \mu \mathrm{L}$ (reference range, 150,000-350,000), and D-dimer was high, between 13 and $142 \mu \mathrm{g} / \mathrm{mL}$ (reference range: $<0.5 \mu \mathrm{g} / \mathrm{mL}$ ). When performed, a platelet factor 4 (PF4)heparin enzyme-linked immunosorbent assay (ELISA) was positive, with high optical densities for most patients. In a functional assay using washed platelets, these antibodies showed platelet activation that was mostly dependent on the addition of PF4 via Fc gamma-receptor (FcrRIIA). Platelet activation was also reported in the presence of the vaccine. Some authors proposed a potential diagnostic strategy for suspected VITT, ${ }^{1}$ which includes immunoassay screening. Since we and others have demonstrated before that significant differences exist between different PF4/heparin immunoassays on the market, ${ }^{5-7}$ we initiated an interlaboratory comparison.

\section{Methods}

\section{Blood Samples and Sample Distribution}

Blood samples were from 12 patients who became symptomatic with low platelet counts, elevated D-dimer, and signs or symptoms of thrombosis within 21 days after receiving vaccination against COVID-19 from AstraZeneca (ChAdOx1 nCoV-19 or AZD1222 or Vaxzevria, from Astra-Zeneca, London, United Kingdom). Coagulation parameters, general chemistry, and PF4/heparin immunoassays to exclude heparin-induced thrombocytopenia (HIT) were performed at four different hospitals in Germany. Anonymized leftover material from all patients was tested in hospitals with an established modified heparin-induced platelet aggregation (HIPA) assay. Subsequently, blinded samples were distributed to all participants who performed their local PF4/heparin immunoassays.

\section{Modified Heparin-Induced Platelet Aggregation}

HIPA tests were performed as described before, with minor modifications. ${ }^{8}$ Serum was tested with washed platelets from four different healthy donors in the absence (buffer alone) or in the presence of heparin $(0.2$ and $100 \mathrm{IU} / \mathrm{mL})$. In additional studies, platelets were preincubated for 10 minutes with PF4 $(25 \mu \mathrm{g} / \mathrm{mL}$ [Chromatec, Greifswald, Germany]). Wells were examined optically at 5-minute intervals for loss of turbidity. A serum was considered reactive (positive) if a shift from turbidity to transparency occurred within 30 minutes in at least two platelet suspensions. Observation time was 45 minutes. Each test included a diluted serum from a patient with HIT as a weak positive control, collagen $(5 \mu \mathrm{g} / \mathrm{mL}$ [Collagen Horn, Takeda, Linz, Austria]) as strong positive control, and a serum from a healthy donor as a negative control.

\section{Enzyme-Linked Immunosorbent Assays}

ELISA no. 1 was Lifecodes PF4 immunoglobulin G (IgG) from Immucor (Waukesha, United States), and ELISA no. 2 was ZYMUTEST HIA, IgG from Hyphen (Neuville-sur-Oise, France). Both assays were used according to the manufacturers' instructions. For Lifecodes PF4 IgG, a sample was considered reactive if optical density (OD) was 0.40 or greater. For Hyphen ZYMUTEST HIA, IgG, a sample was considered reactive if OD was $>0.30$ (0.300-0.499 "gray zone," $\geq 0.500$ positive).

\section{Rapid Immunoassays}

The particle gel immunoassay (PaGIA) is commercially available as ID-PaGIA heparin/PF4 antibody test (Bio-Rad, Hercules, United States). Patient serum is added with heparin/PF4 complexes bound to red, high-density polystyrene particles on a plastic card with gel columns. Antiheparin/PF4 antibodies bind to antigen-coated beads, which agglutinate. After centrifugation, agglutinated beads remain on the top of the gel column, whereas nonagglutinated beads become visible at the bottom. PaGIA results were judged by two independent observers.

The lateral flow assay (LFA) was from Milenia Biotec (Giessen, Germany). The test is commercially available as Milenia QuickLine HIT-Test. It was used as previously described. ${ }^{9}$ Briefly, antibodies are detected based on the principle of capillary action, which induces a flow of the test sample along a solid phase (test strip). Patient serum and reagent are added to the flow system. The reagent contains ligand-labeled human PF4 in complex with a polyanion. A positive reaction becomes visible as an intensively colored line.

An IgG-chemiluminescence immunoassay (CLIA), commercially available as HemosIL AcuStar HIT-IgG (PF4-H), was from Instrumentation Laboratory (IL, Bedford, Massachusetts, United States). The assay was used according to the manufacturer's instructions on an automated ACL AcuStar Hemostasis Testing System (IL). In this assay, anti-PF4/heparin antibodies bind to magnetic particles coated with PF4/ polyvinyl sulfonate. Results were classified as negative $(0.00-0.99 \mathrm{U} / \mathrm{mL})$ or positive $(\geq 1.00 \mathrm{U} / \mathrm{mL})$.

\section{Extended Testing}

To exclude false-positive results for ELISA, a heparin inhibition step was performed. A final concentration of $100 \mathrm{IU}$ heparin/mL was added to the sample, and reduction in OD with and without heparin was calculated. Any reduction above $50 \%$ was considered to demonstrate PF4/heparin complex specificity. To exclude false-negative reactions 
Table 1 VITT patient characteristics

\begin{tabular}{|l|l|l|l|l|l|}
\hline Patient no. & $\begin{array}{l}\text { Time from } \\
\text { vaccination to } \\
\text { admission (d) }\end{array}$ & $\begin{array}{l}\text { Platelet count } \\
\text { at admission } \\
\text { (cells/ } \mathbf{\mu L})\end{array}$ & $\begin{array}{l}\text { D-dimer at } \\
\text { admission } \\
(\boldsymbol{\mu g} / \mathbf{m L})\end{array}$ & $\begin{array}{l}\text { Macrovascular } \\
\text { thrombosis }\end{array}$ & $\begin{array}{l}\text { Heparin prior } \\
\text { to admission }\end{array}$ \\
\hline 1 & 7 & 56,000 & 9 & DVT, PE & No \\
\hline 2 & 10 & 22,000 & NA & CVST, PE & No \\
\hline 3 & 8 & 87,000 & 10 & No & No \\
\hline 4 & 6 & 40,000 & NA & CVST & No \\
\hline 5 & 11 & 27,000 & $>35$ & CSVT & No \\
\hline 6 & 8 & 40,000 & $>35$ & Stroke & No \\
\hline 7 & 5 & 105,000 & 22 & No & No \\
\hline 8 & 9 & 60,000 & $>35$ & CVST & No \\
\hline 9 & 20 & 71,000 & NA & PE & No \\
\hline 10 & 11 & 10,000 & $>35$ & CVST & No \\
\hline 11 & 9 & 8,000 & $>35$ & CVST & No \\
\hline 12 & 9 & 18,000 & NA & PE & No \\
\hline
\end{tabular}

Abbreviations: CVST, cerebral venous sinus thrombosis; DVT, deep vein thrombosis; NA, not available; PE, pulmonary embolism; VITT, vaccineinduced thrombotic thrombocytopenia.

${ }^{\mathrm{a}}$ Diagnosed with transient ischemic attack (TIA).

because of high-dose hook (prozone) effects, three samples (no. 1, 2, and 3) were diluted 1:10 and 1:50 and retested by ELISA, PaGIA, and LFA. Diluted samples were also used for the CLIA assay despite the fact that the assay procedure already contains an additional wash step to exclude high-dose hook (prozone) effects.

\section{Results}

Characterization of sera: A total of 12 eligible patients were identified. Patient characteristics are summarized in - Table 1. In a first attempt to appropriately characterize this material, sera from all patients were tested in a modified HIPA by two laboratories ( - Table 2). In 10/12 samples, serum alone was able to activate test platelets. One serum (no.6) did not activate platelets, and in one (no. 7) results were discrepant between the two laboratories. All reactions were inhibited in the presence of heparin $100 \mathrm{U} / \mathrm{mL}$. All 12 sera gave positive reactions in the modified HIPA with addition of PF4.

In the reaction in presence of $0.2 \mathrm{U}$ heparin $/ \mathrm{mL}, 4$ sera gave positive results, 3 gave borderline results, and 5 gave negative results. Concordance between the two laboratories was very good with no differences between them for 10 sera (83\%) and with only minor differences not affecting the interpretation of results for 2 sera (17\%).

Based on HIPA results, 8 sera were characterized as typical for VITT: positive buffer reaction, negative reaction in presence of $0.2 \mathrm{U} / \mathrm{mL}$ heparin, negative (or borderline) reaction in presence of $100 \mathrm{U} / \mathrm{mL}$ heparin, and positive in presence of PF4 (sera no. 1-3, and no. 6-10). Serum no. 6 differed slightly from the other seven sera since it was nonreactive in the buffy only test. Note that for all sera with a positive reaction in presence of buffer, the median reaction time was strongly reduced when PF4 was present (laboratory A, 20 vs. 7.5 minutes; $p<0.05$, Wilcoxon signed-ranks test; and laboratory B, 15 vs. 7.5 minutes, $p<0.05$, Wilcoxon signed-ranks test). Four sera (no. $4,5,11$, and 12) gave positive results in the presence of $0.2 \mathrm{U}$ heparin $/ \mathrm{mL}$. In contrast to $\mathrm{PF} 4,0.2 \mathrm{U} / \mathrm{mL}$ heparin appears to prolong the reaction time in the assay, but there were exceptions (serum no. 11), and the overall number of sera reactive with $0.2 \mathrm{U} / \mathrm{mL}$ heparin in this study was too low for statistical evaluation. Again, based on HIPA results only, the 4 sera were characterized as VITT/HIT, since no definite differentiation between the two types of immune thrombotic thrombocytopenia could be made. However, none of the patients was exposed to heparin before admission to hospital, excluding HIT based on clinical criteria.

Results obtained in immunoassays: Five different immunoassays are in use in the four laboratories. Two laboratories use "rapid assays" (PaGIA or LFA) for emergency samples, which they confirm by ELISA testing; one laboratory uses ELISA only; and one laboratory uses CLIA only. Samples were run in each test, and results are summarized in - Table 3. ELISA technology identified $12 / 12(100 \%)$ or $11 / 12$ (92\%) VITT sera. Only three VITT samples gave positive or borderline reactions in the PaGIA (25\%). The one serum with a borderline result in PaGIA also gave a borderline result in LFA (8\%), whereas the other 11 sera were negative. Ten samples were tested by CLIA, all of which were nonreactive.

Extended testing: To confirm the specificity of results obtained by ELISA, two laboratories included an inhibition step with $100 \mathrm{U}$ heparin/mL. Inhibition of more than $50 \%$ of the OD was seen for all samples (100\%), confirming the specificity of the ELISA results. To exclude false-negative reactions because of potential high-dose hook effects, samples no. 1,2 , and 12 were diluted $1: 10$ and $1: 50(\mathrm{v} / \mathrm{v})$ in saline and retested by PaGIA, LFA, and CLIA. None of the diluted sera 
Table 2 HIPA testing and laboratory diagnosis based on HIPA results

\begin{tabular}{|c|c|c|c|c|c|c|c|c|c|c|}
\hline \multirow[t]{3}{*}{ Patient no. } & \multicolumn{8}{|c|}{ HIPA in presence of ... } & \multirow[t]{3}{*}{ Concordance } & \multirow{3}{*}{$\begin{array}{l}\text { Laboratory } \\
\text { diagnosis } \\
\text { based on } \\
\text { HIPA results }\end{array}$} \\
\hline & \multicolumn{2}{|c|}{ Buffer only } & \multicolumn{2}{|c|}{$\begin{array}{l}\text { Buffer plus } \\
\text { heparin } \\
0.2 \mathrm{U} / \mathrm{mL}\end{array}$} & \multicolumn{2}{|c|}{$\begin{array}{l}\text { Buffer plus } \\
\text { heparin } \\
100 \mathrm{U} / \mathrm{mL}\end{array}$} & \multicolumn{2}{|c|}{$\begin{array}{l}\text { Buffer plus } \\
\text { PF4 } \\
25 \mu \mathrm{g} / \mathrm{mL}\end{array}$} & & \\
\hline & A & B & A & B & $A$ & B & $A$ & B & & \\
\hline 1 & 20 & 15 & - & - & - & - & 15 & 10 & ++ & VITT \\
\hline 2 & 10 & 15 & - & - & - & - & 5 & 5 & ++ & VITT \\
\hline 3 & 5 & 5 & - & - & - & - & 5 & 5 & ++ & VITT \\
\hline 4 & 5 & 5 & 20 & 30 & - & - & 5 & 5 & ++ & VITT/HIT \\
\hline 5 & 30 & 30 & 30 & 30 & - & - & 30 & 25 & ++ & VITT/HIT \\
\hline 6 & - & - & - & - & - & - & 30 & 30 & ++ & VITT \\
\hline 7 & 30 & $B L$ & - & - & - & - & 10 & 5 & + & VITT \\
\hline 8 & 25 & 25 & - & $\mathrm{BL}$ & - & - & 5 & 10 & + & VITT \\
\hline 9 & 25 & 15 & $\mathrm{BL}$ & $B L$ & - & - & 10 & 10 & ++ & VITT \\
\hline 10 & 25 & 30 & $\mathrm{BL}$ & $\mathrm{BL}$ & - & - & 15 & 15 & ++ & VITT \\
\hline 11 & 5 & 15 & 5 & 5 & - & - & 5 & 5 & ++ & VITT/HIT \\
\hline 12 & 5 & 15 & 15 & 30 & - & - & 5 & 5 & ++ & VITT/HIT \\
\hline $\begin{array}{l}\text { Median time } \\
\text { to aggregation }\end{array}$ & 20 & 15 & $\begin{array}{l}17 .- \\
5\end{array}$ & 30 & - & - & 7.5 & 7.5 & & \\
\hline
\end{tabular}

Abbreviations: HIPA, heparin-induced platelet aggregation; HIT, heparin-induced thrombocytopenia; PF4, platelet factor 4; VITT, vaccine-induced thrombotic thrombocytopenia.

Note: A sample was considered positive if reactive with at least two out of four platelet suspensions within 30 minutes. Median reaction times for this table were calculated as the median for all reactive cells per analysis. A, B denotes the two test laboratories performing HIPA. BL, borderline result (aggregation observed at $t=45$ minutes, but not within the first 30 minutes of the assay). "-“ indicates a negative test result (no aggregation at $t=45$ minutes). Concordance between the two laboratories was rated as follows: ++ no difference, + difference not affecting interpretation, 0 difference possibly affecting interpretation, - difference affecting interpretation.

became reactive, excluding a high-dose hook effect as potential explanation for false-negative test results.

\section{Discussion}

Here we report the results of an interlaboratory comparison on VITT laboratory diagnosis, which involved four laboratories. We could demonstrate that only PF4/heparin tests based on ELISA technology, but none of the other PF4/heparin assays, are suitable to detect VITT antibodies. We could also show that between two of the laboratories, functional testing of VITT antibodies by modified HIPA revealed comparable results.

In the functional characterization of the serum material, we were able to reproduce data published recently. ${ }^{1}$ All sera led to variable, but mostly positive platelet activation with buffer only, and variable, but mostly negative results with low-dose heparin. In contrast, strong platelet activation was seen in the presence of PF4, while high-dose heparin inhibited platelet activation. In the majority of sera reactive with buffer, addition of PF4 reduced the median reaction time in the functional test. Some sera were reactive with low-dose heparin; blinded against clinical information, we felt unable to differentiate between VITT and HIT by HIPA for these sera. However, none of the patients received heparin before the sample was taken, and all patients were vaccinated within the last 5 to 20 days prior to hospital admission. The concordance rate between the two laboratories was within the range expected for functional testing. ${ }^{10}$

All samples produced high signals in both ELISA assays with one exception. Serum no. 8 was reactive in the Immucor assay, but not in the Hyphen assay. Such minor differences in sensitivity were also reported for HIT antibodies in the past. ${ }^{11}$ In general, sensitivity of ELISA assays was satisfactory (92-100\%).

In contrast, laboratories using PaGIA, LTA, or CLIA as first test, were unable to identify VITT antibodies. Even if considered that borderline results in one of these assays would have led to further testing, sensitivity of PaGIA (25\%) and LFA (8\%) was far too low. Whether observed borderline results in PaGIA were indicative of (weak) specific reactions or simply mirror the previously reported low test specificity, ${ }^{12}$ remains unclear.

None of the samples was reactive in the CLIA assay. This is a very interesting observation, because it may indicate that VITT could possibly be diagnosed by a combination of two tests. HIT antibodies are usually reactive with all PF4/heparin assays, with minor differences in sensitivity, and more relevant differences in specificity. ${ }^{11}$ CLIA has been shown to be highly sensitive and specific for HIT antibodies. ${ }^{13}$ VITT antibodies were nonreactive in the patients reported here. Accordingly, a positive ELISA in connection with a negative CLIA appears to be indicative of VITT, although caution 
Table 3 Results obtained in PF4/heparin immunoassays

\begin{tabular}{|l|l|l|l|l|l|l|}
\hline Patient no. & $\begin{array}{l}\text { Laboratory } \\
\text { diagnosis based on HIPA }\end{array}$ & $\begin{array}{l}\text { ELISA 1 } \\
\text { (OD) }\end{array}$ & $\begin{array}{l}\text { ELISA 2 } \\
\text { (OD) }\end{array}$ & PaGIA & CLIA & LFA \\
\hline 1 & VITT & 2.6 & 2.7 & Neg & 0.02 & Neg \\
\hline 2 & VITT & 2.9 & 3.1 & Pos & 0.27 & Neg \\
\hline 3 & VITT & 3.1 & 3.6 & Neg & 0.63 & Neg \\
\hline 4 & VITT/HIT & 2.7 & 2.1 & BL & NA & BL \\
\hline 5 & VITT/HIT & 2.2 & 1.6 & Neg & 0.02 & Neg \\
\hline 6 & VITT & 2.6 & 2.7 & Neg & 0.16 & Neg \\
\hline 7 & VITT & 2.7 & 2.1 & Neg & 0.07 & Neg \\
\hline 8 & VITT & 2.1 & 0.24 & Pos & NA & Neg \\
\hline 9 & VITT & 2.8 & 1.7 & Neg & 0.01 & Neg \\
\hline 10 & VITT & 2.7 & 2.1 & Neg & 0.06 & Neg \\
\hline 11 & VITT/HIT & 2.9 & 3.1 & Neg & 0.04 & Neg \\
\hline 12 & VITT/HIT & 3.6 & 3.7 & Neg & 0.13 & Neg \\
\hline Sensitivity (\%) & & 100 & 91.6 & 0.25 & 0 & 0.08 \\
\hline
\end{tabular}

Abbreviations: BL, borderline result; CLIA, chemiluminescence immunoassay; ELISA, enzyme-linked immunosorbent assay; HIPA, heparin-induced platelet aggregation; HIT, heparin-induced thrombocytopenia; LFA, lateral flow assay; NA, not available; Neg, negative test result; OD, optical density; PaGIA, particle gel immunoassay; PF4, platelet factor 4; Pos, positive test result; VITT, vaccine-induced thrombotic thrombocytopenia.

should be exercised to generalize this observation before higher numbers of patients have been studied. Since both assay types are commercially available, such a combined test strategy may help establishing a diagnosis of VITT in the absence of functional (in-house) testing.

It is currently unknown how exactly epitopes recognized by VITT antibodies differ from those recognized by HIT antibodies. Strongly positively charged PF4 forms antigenic complexes with a variety of polyanions including polyphosphates, nucleic acids, glycosaminoglycans, and sulfated anticoagulants. ${ }^{14}$ Binding of anti-PF4/heparin antibodies in HIT is dependent on conformational changes in PF4, which are induced by the binding partner heparin. ${ }^{15}$ The binding partner of PF4 in VITT is still unclear, but a conformational change of PF4 which induces a different antigen complex can be assumed.

The Immucor ELISA uses polyvinyl sulfonate and PF4, and the Hyphen ELISA uses protamine sulfate, unfractionated heparin, and platelet lysate containing PF4 and other chemokines to detect HIT antibodies. Apparently, both ELISA assays provide epitopes for both, VITT antibodies and HIT antibodies. Polyvinyl sulfonate and PF4 attached to magnetic particles are also used for the CLIA, which, however, did not detect VITT antibodies in our series. Differences between the CLIA and the ELISA that could affect binding of VITT antibodies include the presence of additional polar groups on polystyrol microtiter plates used for ELISA, and the availability of PF4 in higher amounts. The latter would be in accordance with the observation that addition of PF4 greatly enhances the detectability of VITT antibodies in the functional assay. ${ }^{1}$

Despite this uncertainty about the true epitope of VITT antibodies, we have clearly demonstrated that, as a single test, LFA, PaGIA, or CLIA are unsuitable for the detection of VITT antibodies.
Accordingly, as a single test, these assays cannot be incorporated in a diagnostic algorithm for suspected VITT. In contrast, a combination of PF4/heparin ELISA and CLIA may be useful to establish the laboratory diagnosis of VITT in the absence of functional in-house assays. However, comparing functional test performance between two laboratories at least indicates that establishing functional VITT testing is feasible.

\section{What is known about this topic?}

- COVID-19 vaccine ChAdOx1 nCov-19 may lead to vaccine-induced thrombotic thrombocytopenia (VITT).

- The diagnostic approach includes anti-PF4/heparin assays for the detection of VITT antibodies, but the sensitivity of different assays is unknown.

\section{What does this paper add?}

- In an interlaboratory comparison, we show that only PF4/heparin ELISA, but no other immunological assay, detect VITT antibodies reliably.

- We demonstrate that "rapid" PF4/heparin assays are unsuitable in the diagnostic workup of suspected VITT and must be avoided.

\section{Author Contributions}

U.J.S., A.T., and K.A. designed the study, analyzed the data, and wrote the manuscript. N.C., A.C., J.M., B.P., and A.T. performed laboratory studies and analyzed data. All authors provided relevant material. All authors read and approved the manuscript. 


\section{Conflict of Interest}

None declared.

\section{Acknowledgments}

We would like to acknowledge the excellent technical support from Marica Müller and Astrid Giptner (Giessen, Germany).

\section{References}

1 Greinacher A, Thiele T, Warkentin TE, Weisser K, Kyrle PA, Eichinger S. Thrombotic thrombocytopenia after ChAdOx1 nCov-19 vaccination. N Engl J Med 2021;384(22):2092-2101

2 Schultz NH, Sørvoll IH, Michelsen AE, et al. Thrombosis and thrombocytopenia after ChAdOx1 nCoV-19 vaccination. N Engl J Med 2021;384(22):2124-2130

3 Tiede A, Sachs UJ, Czwalinna A, et al. Prothrombotic immune thrombocytopenia after COVID-19 vaccine. Blood 2021: blood.2021011958

4 Wolf ME, Luz B, Niehaus L, Bhogal P, Bäzner H, Henkes H. Thrombocytopenia and intracranial venous sinus thrombosis after "COVID-19 vaccine AstraZeneca" exposure. J Clin Med 2021;10(08):1599

5 Nagler M, Bachmann LM, ten Cate H, ten Cate-Hoek A. Diagnostic value of immunoassays for heparin-induced thrombocytopenia: a systematic review and meta-analysis. Blood 2016;127(05): 546-557

6 Althaus K, Westphal A, Strobel U, Bakchoul T, Greinacher A. Reallife evaluation of an automated immunoassay for diagnosis of heparin-induced thrombocytopenia. Thromb Res 2020;196: 400-403
7 Bankova A, Andres Y, Horn MP, Alberio L, Nagler M. Rapid immunoassays for diagnosis of heparin-induced thrombocytopenia: comparison of diagnostic accuracy, reproducibility, and costs in clinical practice. PLoS One 2017;12(06):e0178289

8 Greinacher A, Michels I, Kiefel V, Mueller-Eckhardt C. A rapid and sensitive test for diagnosing heparin-associated thrombocytopenia. Thromb Haemost 1991;66(06):734-736

9 Sachs UJ, von Hesberg J, Santoso S, Bein G, Bakchoul T. Evaluation of a new nanoparticle-based lateral-flow immunoassay for the exclusion of heparin-induced thrombocytopenia (HIT). Thromb Haemost 2011;106(06):1197-1202

10 Eekels JJM, Althaus K, Bakchoul T, et al. An international external quality assessment for laboratory diagnosis of heparin-induced thrombocytopenia. J Thromb Haemost 2019;17(03):525-531

11 Vianello F, Sambado L, Scarparo P, et al. Comparison of three different immunoassays in the diagnosis of heparin-induced thrombocytopenia. Clin Chem Lab Med 2015;53(02):257-263

12 Marchetti M, Barelli S, Zermatten MG, et al. Rapid and accurate Bayesian diagnosis of heparin-induced thrombocytopenia. Blood 2020;135(14):1171-1184

13 Warkentin TE, Sheppard JI, Linkins L-A, Arnold DM, Nazy I. High sensitivity and specificity of an automated IgG-specific chemiluminescence immunoassay for diagnosis of HIT. Blood 2018;132 (12):1345-1349

14 Cines DB, Yarovoi SV, Zaitsev SV, et al. Polyphosphate/platelet factor 4 complexes can mediate heparin-independent platelet activation in heparin-induced thrombocytopenia. Blood Adv 2016;1(01):62-74

15 Kreimann M, Brandt S, Krauel K, et al. Binding of anti-platelet factor 4/heparin antibodies depends on the thermodynamics of conformational changes in platelet factor 4. Blood 2014;124(15): 2442-2449 